\title{
Migraine with Visual Aura a Risk Factor for Incident Atrial Fibrillation
}

Souvik Sen MD, MS, MPH, X Michelle Androulakis MD, MS, Viktoriya Duda MPH, Alvaro Alonso MD PhD, Lin Yee Chen MD, Elsayed Z. Soliman MD, Jared Magnani MD, MSc, Tushar Trivedi MD, Anwar T. Merchant DMD, ScD, Rebecca F. Gottesman MD PhD, Wayne D Rosamond, PhD, MS

\section{INTRODUCTION}

Epidemiological studies have shown that migraine with aura is associated with increased risk of stroke based on meta-analysis of diverse cohorts of patients (Risk Ratio 2.14, Cl 95\%, 1.333.43). [Hu X Neurosci 2017; 38:33-40] Migraine with visual aura was associated with an increased risk of stroke or transient ischemic stroke (TIA) symptoms and ischemic stroke events in the Atherosclerosis Risk in Community (ARIC) study cohort. [Stang PE et al Neurology 2005; 64:1573-7] We recently reported that migraine with visual aura is independently associated with increased risk of cardioembolic stroke compared with migraine without aura. [Androulakis XM Neurology 2016; 87:2527-32] Since AF is a common source of cardioembolic stroke, the question that begs to be answered is if the association between migraine with visual aura and cardioembolic stroke may be explained by a higher rate of atrial fibrillation (AF) in this subgroup of the cohort. [Sposato LA Neurology 2016; 87:2504-5] Therefore, we proposed to evaluate the hypothesis of potential association between migraine with visual aura and incident AF.

\section{METHODS}

The ARIC study established a cohort to analyze atherosclerosis causes and clinical outcomes. [ARIC Investigators Am J Epidemiol 1989; 129:687-702] A total of 15,792 participants age 45-64 at time of enrollment. Participants came from 4 different US communities. participants who completed visit 3 (1993$1995)$ in the ARIC study $(n=12,882)$ were included in our study. Participants with known history of AF or those with inadequate/missing AF information prior to visit $3(n=819)$ those with prior ischemic stroke $(\mathrm{N}=87)$ as well as any participants with missing headache information $(n=37)$ were excluded. After excluding these subjects, a total of 11,939 participants fulfilled selection criteria and their data was included in study analysis.

Assessment of migraine: As part of the participants' headache assessment, a headache questionnaire was administered during visit 3. Modified International Classification of Headache Disorders (ICHD III beta) [Androulakis XM Neurology 2016; 87:2527-32] diagnostic criteria were used to differentiate migraine with visual aura and migraine without visual aura based on the data collected in this questionnaire.

AF Ascertainment: Diagnoses of AF in ARIC study participants were sourced from study performed electrocardiograms (ECG), hospital discharge codes, and death certificates. [Alonso A 2009 Am Heart J 2009; 158:111-7]

\section{RESULTS}

Migraines with visual aura $(\mathrm{N}=426)$, migraine without visual aura $(\mathrm{N}=1090)$, non-migraine headache $(\mathrm{N}=1,018)$ and no headache $(\mathrm{N}=9,405)$. 20-year follow-up period incident $\mathrm{AF}$ was noted in 232 $(15 \%) / 1,516$ with migraine, 142 (14\%) /1,018 with non-migraine headache and 1,623 (17\%) /9,405 without headache. After adjustment for confounders, migraine with visual aura was associated with increased risk of AF compared to no headache (HR 1.30, 95\% Cl 1.03-1.62) and to migraine without visual aura (HR 1.39, 95\% Cl 1.05-1.83).

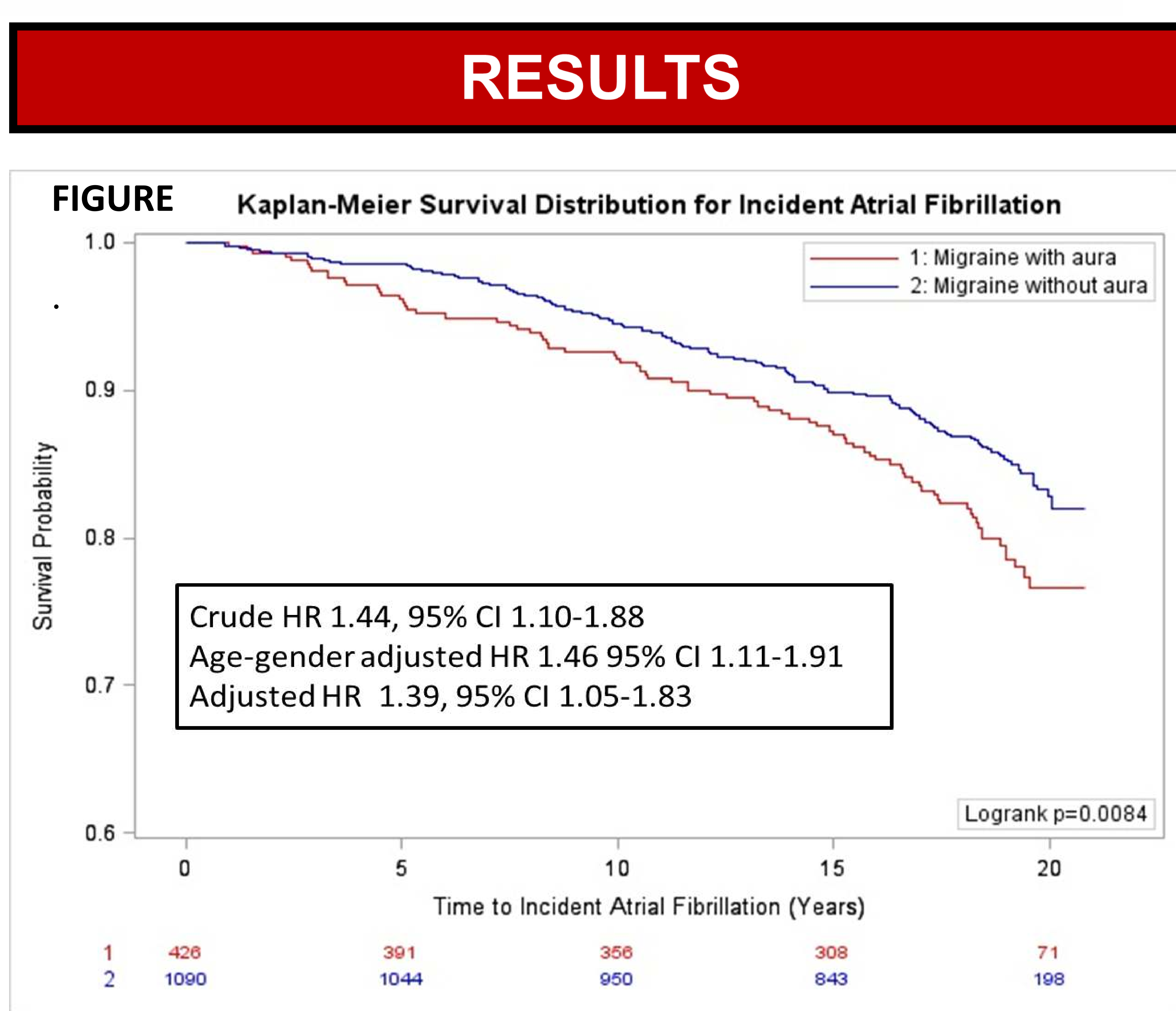

TABLE

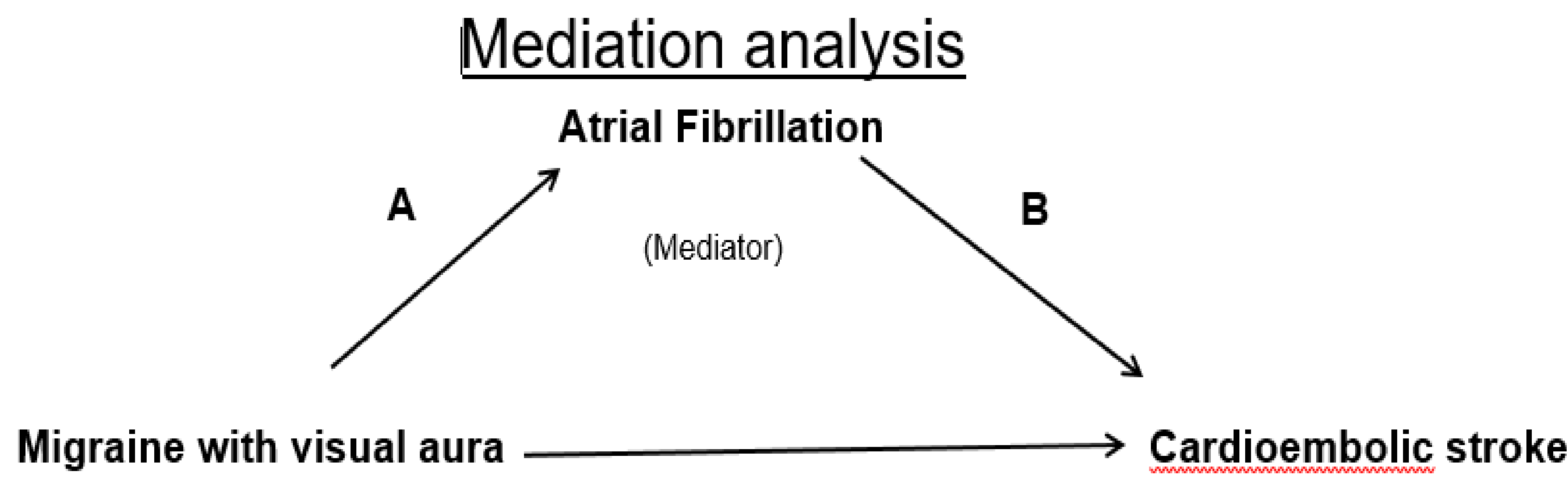

(Exposure)

$\mathrm{C} / \mathbf{C}^{\prime}$

(Outcome)

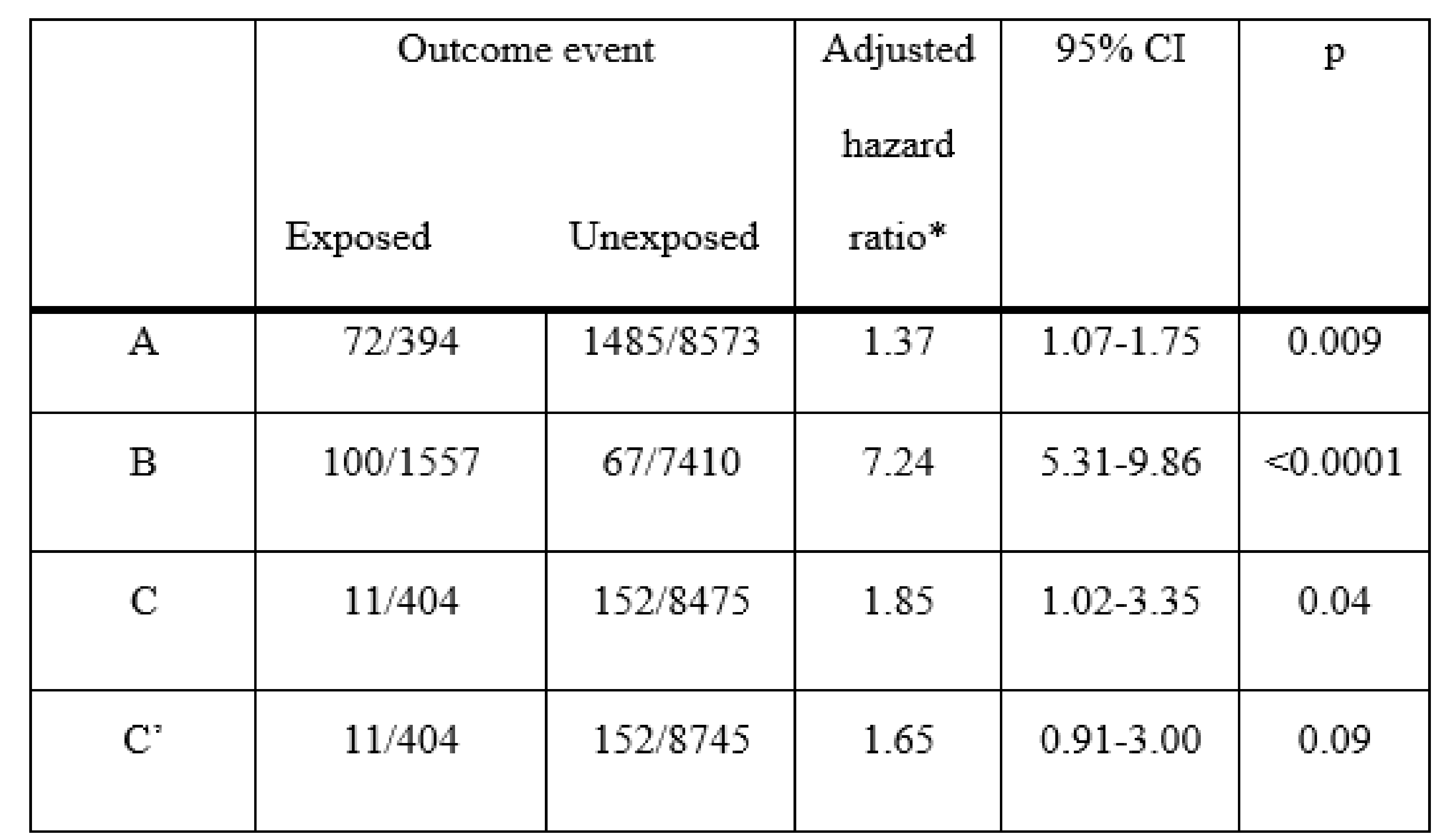

${ }^{*}$ Adjusted for age and sex

A: Total effect of migraine with visual aura on atrial fibrillation

B: Total effect of atrial fibrillation on cardioembolic stroke additionally adjusted for migraine with visual aura

C: Total effect of migraine with visual aura on cardioembolic stroke C': Total effect of migraine with visual aura on cardioembolic stroke additionally adjusted for atrial fibrillation

\section{CONCLUSION}

Migraine with aura was associated with increased risk of AF. The migraine with aura associated $A F$, may potentially lead to ischemic strokes. 